\title{
Photochemical Organocatalytic Borylation of Alkyl Chlorides, Bromides, and Sulfonates
}

\author{
Daniele Mazzarella, ${ }^{\dagger, \nabla}$ Giandomenico Magagnano, ${ }^{\dagger, \nabla}$ Bertrand Schweitzer-Chaput, ${ }^{\dagger \oplus}$ \\ and Paolo Melchiorre $*,+, \$ \odot$
}

${ }^{\dagger}$ ICIQ-Institute of Chemical Research of Catalonia, The Barcelona Institute of Science and Technology, Avinguda Països Catalans 16, 43007 Tarragona, Spain

${ }^{\ddagger}$ ICREA, Passeig Lluís Companys 23, 08010 Barcelona, Spain

${ }^{\S}$ IIT-Istituto Italiano di Tecnologia, via Morego 30, 16163 Genoa, Italy

\section{Supporting Information}

ABSTRACT: Reported herein is a photochemical strategy for the borylation of alkyl halides using bis(catecholato)diboron as the boron source. This method exploits the ability of a nucleophilic dithiocarbonyl anion organocatalyst to generate radicals via an $\mathrm{S}_{\mathrm{N}} 2$-based photochemical catalytic mechanism, which is not reliant on the redox properties of the substrates. Therefore, it grants access to alkyl boronic esters from readily available but difficult-to-reduce electrophiles, including benzylic and allylic chlorides, bromides, and mesylates, which were inert to or unsuitable for previously reported metal-free borylation protocols.

KEYWORDS: borylation, organocatalysis, photochemistry, radicals, $S_{N} 2$ chemistry

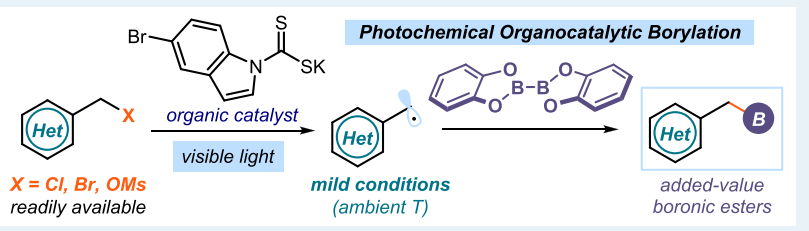

$\mathrm{B}$ oronic acids and esters are extensively used in material science, ${ }^{1}$ chemosensoring, ${ }^{2}$ and medicinal chemistry. ${ }^{3}$ They are also extremely valuable synthetic precursors that can be readily transformed to a variety of useful functional groups. ${ }^{4}$ For example, the prominence of the Suzuki-Miyaura reaction ${ }^{4 a}$ has established the crucial synthetic importance of aromatic boronic acids and esters. Therefore, there are welldeveloped synthetic protocols for making aromatic derivatives. $^{5}$

Alkyl boronic acids and esters are also highly useful intermediates, ${ }^{6}$ which justifies the recent interest in developing effective methods for their preparation. Traditionally, they have been accessed via the hydroboration of alkenes or from organo-lithium and organo-magnesium reagents via transmetalation. ${ }^{5}$ Both strategies suffer from regioselectivity issues and functional group incompatibility. A more effective approach relies on the transition-metal-catalyzed borylation of alkyl halides with diborons (Figure 1a, left arrow). While most resulting strategies require the use of alkyl bromides or iodides, $^{7}$ a few catalytic systems based on manganese ${ }^{8 a}$ and copper $^{8 b}$ have been described for the borylation of unactivated alkyl chlorides. These protocols, although effective, require the use of transition-metal catalysts, which might be incompatible with $N$-heterocycle-containing substrates because of deleterious heteroatom-catalyst binding effects. Indeed, $N$-heterocyclic moieties, which are common motifs in drug molecules, generally represent a significant tolerability challenge for synthetic methods. ${ }^{9}$ As a result, few metal-based borylation methods compatible with heteroarenes containing multiple

Figure 1. (a) Previous catalytic borylation strategies require either the use of metals (left panel), which complicates the compatibility with $\mathrm{N}$-heterocycle-containing substrates, or of radical precursors containing redox active moieties and alkyl iodides (right panel). (b) The photochemical strategy presented herein uses a nucleophilic organic catalyst and visible light for the borylation of redox-inert alkyl electrophiles, including chlorides. It is compatible with $\mathrm{N}$-heterocyclic moieties.

heteroatoms and high nitrogen content have been reported so far. ${ }^{10}$

Received: April 10, 2019

Revised: $\quad$ May 24, 2019

Published: May 28, 2019 
More recent borylation methods have sought to overcome these limitations by using metal-free and functional group tolerant conditions. In this context, radical-mediated processes, in which alkyl radicals are borylated with diboranes, have become increasingly important (Figure 1a, right arrow). ${ }^{11}$ However, these methodologies generally require the use of tailored substrates, purposely adorned with redox-active moieties. ${ }^{12}$ This is because a redox event is needed to trigger the formation of the reactive $\mathrm{C}\left(s p^{3}\right)$-centered radicals. Studer recently developed a metal-free photochemical borylation of alkyl iodides, where light is responsible for radical formation by eliciting homolytic carbon-iodine bond cleavage. ${ }^{13}$ Despite the clear improvement, in terms of atom economy, this borylation process is limited to the use of highly reactive and unstable iodide derivatives.

Here, we report a visible-light-mediated organocatalytic system capable of borylating a wide range of readily available and stable benzylic and allylic chlorides, bromides, and mesylates, which were inert to other metal-free borylation protocols (Figure 1b). Importantly, the method's high functional group tolerance allows us to easily prepare alkyl boronic esters adorned with $N$-heterocycles, which would not be compatible with strong redox-active reagents or metal-based protocols.

This research endeavor was motivated by our interest in using the photochemical reactivity of organocatalytic intermediates to generate radicals under mild conditions. ${ }^{14}$ Specifically, our laboratory ${ }^{15}$ recently reported a visible-lightmediated catalytic strategy that uses a dithiocarbonyl ${ }^{16}$ (DTC) anion A, adorned with an indole chromophoric unit (Figure $2 \mathrm{a})$. This nucleophilic organic catalyst $\mathbf{A}^{17}$ can activate alkyl electrophiles by displacing a variety of leaving groups via an $\mathrm{S}_{\mathrm{N}} 2$ pathway, including chlorides. The resulting photonabsorbing intermediates I afford $\mathrm{C}\left(s p^{3}\right)$-centered radicals upon excitation by visible light and homolytic cleavage of the weak $\mathrm{C}-\mathrm{S}$ bond. ${ }^{18}$ This catalytic $\mathrm{S}_{\mathrm{N}} 2$-based strategy, which is not reliant on the redox properties of the radical precursor, grants access to open-shell intermediates from substrates generally inert to classical radical-generating strategies. ${ }^{19}$

Cognizant of the ability of the commercially available bis(catecholato)diboron $\left(\mathrm{B}_{2} \mathrm{cat}_{2}\right) 2$ to intercept carbon radicals, $^{11-13}$ we wondered if our photochemical strategy could serve for the borylation of readily available alkyl chlorides, bromides, and alcohol derivatives, such as mesylates. As detailed in Figure $2 b$, we envisioned that, upon $\mathrm{S}_{\mathrm{N}} 2$ displacement and photolysis of $\mathrm{I}$, the $\mathrm{C}\left(s p^{3}\right)$-centered radical II could react with $\mathrm{B}_{2} \mathrm{cat}_{2} 2$ to forge a $\mathrm{C}-\mathrm{B}$ bond. The ensuing boron-centered radical IV is then intercepted by dimenthylformamide (DMF) to afford intermediate $\mathbf{V} \cdot{ }^{13} \mathbf{V}$ collapses to afford the target alkyl boronic ester product 3, along with the DMF-complexed boryl radical VI. The open-shell intermediate VI $\left(E_{1 / 2}=-1.53 \mathrm{~V} \text { in } \mathrm{MeCN} \text { vs } S C E\right)^{12 \mathrm{~d}}$ can then perform a single electron reduction of the dithiocarbonyl radical III (the ethyl xanthogenate anion, where $\mathrm{Z}=\mathrm{OEt}$, has a reduction potential of $E_{\text {red }}(\text { III/A) }=+0.04 \mathrm{~V} \text { vs SCE })^{20}$ to close the catalytic cycle and turn over the catalyst $\mathbf{A} .^{21}$

To test the feasibility of our borylation strategy, we selected the commercially available 1 -(chloromethyl)-1H-benzotriazole $\mathbf{1 a}$ as the radical precursor (Table 1). This choice was informed by our desire to develop a metal-free protocol that could (i) activate alkyl chlorides and (ii) tolerate $N$ heterocyclic moieties. The experiments were conducted in $\mathrm{DMF}$ at ambient temperature using 2 equiv of $\mathrm{B}_{2} \mathrm{cat}_{2} 2,10 \mathrm{~mol}$
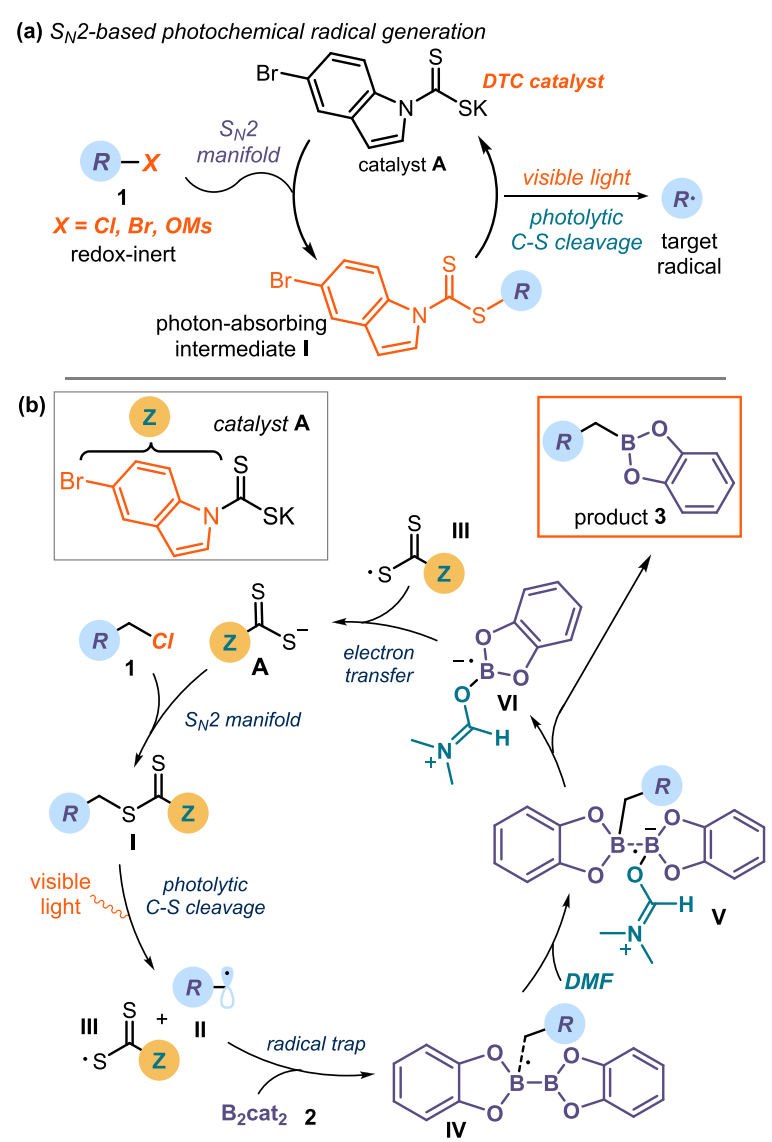

Figure 2. (a) Our recently developed $S_{N} 2$-based method for photochemically generating radicals from alkyl chlorides. (b) Proposed mechanism for the visible-light-driven borylation of alkyl halides $(\mathrm{Z}=$ chromophore $)$.

Table 1. Optimization Studies ${ }^{a}$

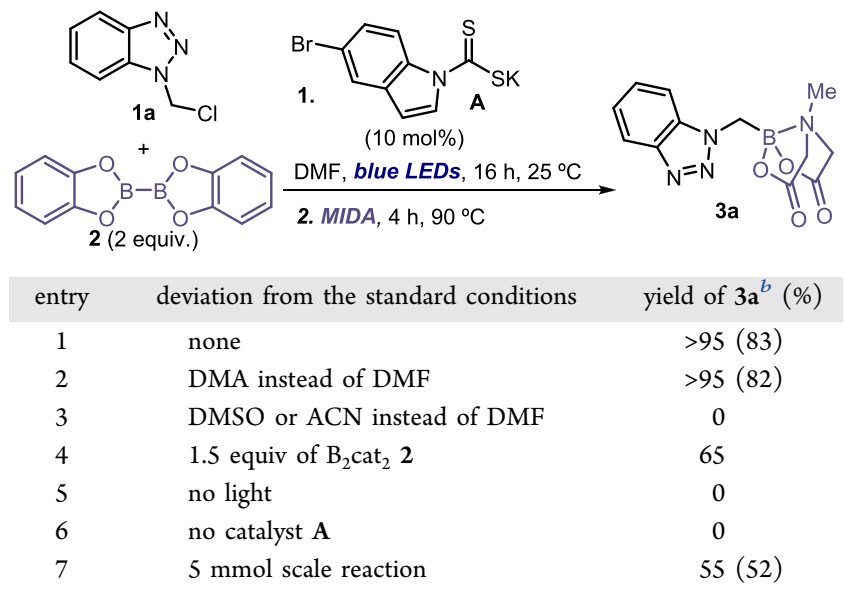

${ }^{a}$ Reactions performed in DMF $(0.4 \mathrm{~mL})$ at ambient temperature for $16 \mathrm{~h}$ on a $0.2 \mathrm{mmol}$ scale under illumination by blue LED strips $\left(\lambda_{\max }\right.$ $=465 \mathrm{~nm})$ and using catalyst $\mathbf{A}(10 \mathrm{~mol} \%)$ and 2 equiv of $2{ }^{b}$ Yield of 3a determined by ${ }^{1} \mathrm{H}$ NMR analysis of the crude mixture using nitromethane as the internal standard; the value shown in parentheses refers to the yield of isolated $\mathbf{3} \mathbf{a}$ after chromatographic purification on silica gel. [Legend: DMF, dimethylformamide; DMA, dimethylacetamide; ACN, acetonitrile; DMSO, dimethyl sulfoxide; MIDA, methyliminodiacetic acid.] 

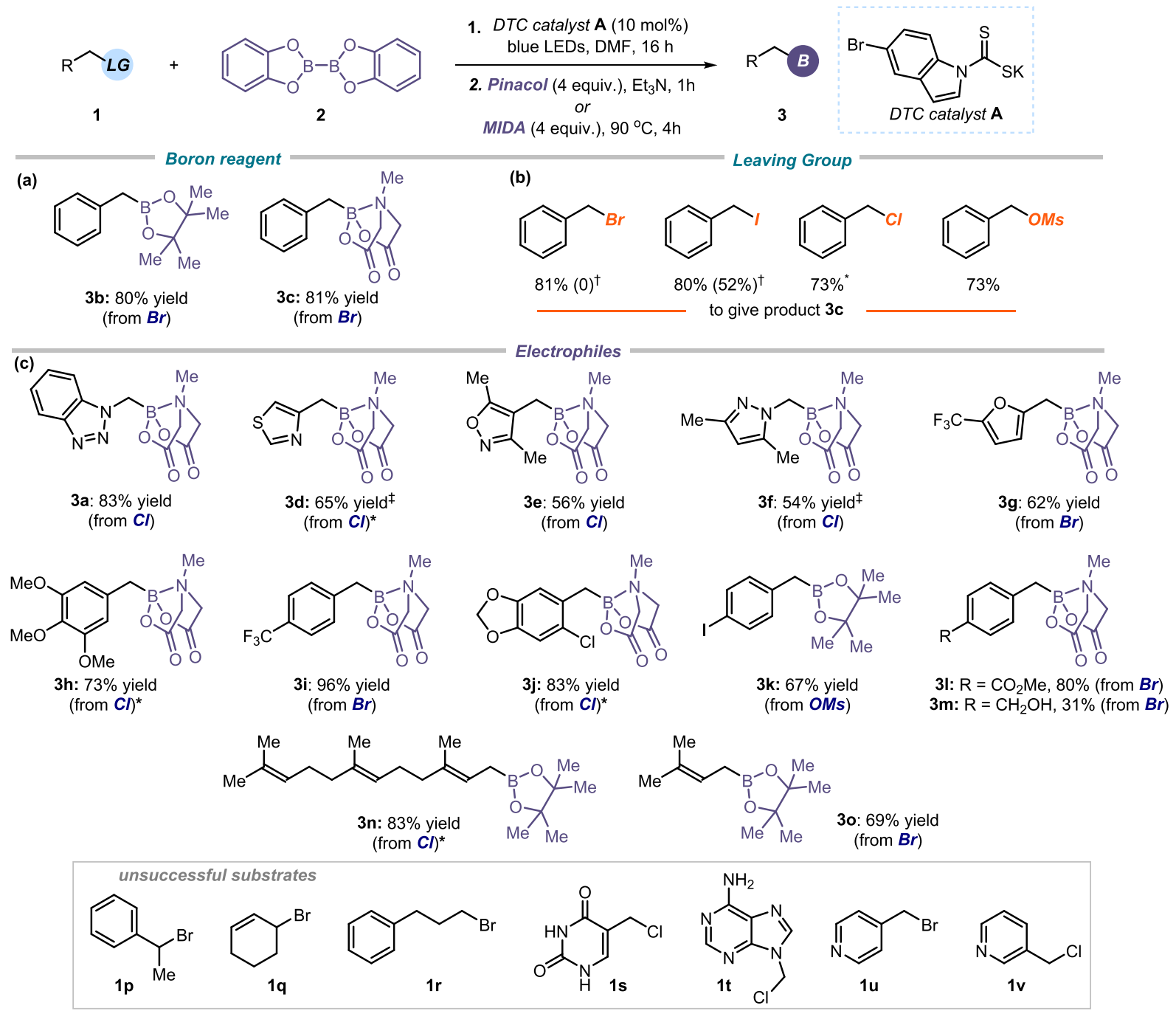

Figure 3. Survey of the electrophiles 1 that can participate in the photochemical borylation reaction. Reactions performed on a $0.2 \mathrm{mmol}$ scale using 2 equiv bis(catecholato)diboron 2 in $0.4 \mathrm{~mL}$ of DMF. Yields of the isolated products 3 are indicated below each entry. [Asterisk symbol (*) denotes 2 equiv of $\mathrm{NaBr}$. Superscripted dagger symbol $(\dagger)$ indicates that the yields in parentheses refer to the reaction performed in the absence of catalyst A. Superscripted double dagger symbol ( $\$)$ denotes the presence of 1 equiv of 2,6-lutidine. MIDA = methyliminodiacetic acid.]

$\%$ of the indole-containing dithiocarbamate catalyst $\mathbf{A}$, and blue LEDs emitting at $465 \mathrm{~nm}$ (see Table 1). After $16 \mathrm{~h}$, the reaction was treated with 4 equiv of methyliminodiacetic acid (MIDA) and heated at $90{ }^{\circ} \mathrm{C}$ to perform ligand exchange and generate the more-stable MIDA boronate 3a. These conditions furnished the borylation product $3 \mathbf{a}$ in high chemical yield (83\%, entry 1 in Table 1$)$.

The use of a different coordinating solvent, such as dimethylacetamide (DMA, entry 2 in Table 1), elicited a similar reactivity, while acetonitrile $(\mathrm{ACN})$ or dimethyl sulfoxide (DMSO) completely inhibited the reaction (Table 1 , entry 3 ). These results are consistent with the need to stabilize the radical intermediate $\mathbf{V}$ (see Figure 2). ${ }^{22}$ Further variations of the initial conditions, including a lower amount of 2 (Table 1, entry 4), led to poorer results. Control experiments established that both catalyst $\mathbf{A}$ and light are required for the reaction to proceed (Table 1, entries 5 and 6). A 25-fold scaleup of the reaction (Table 1 , entry $7,5 \mathrm{mmol}$ scale) delivered product $3 \mathrm{a}$ in $52 \%$ yield $(750 \mathrm{mg})$ using the same standard experimental setup as for the $0.2 \mathrm{mmol}$ process. This experiment indicates that this procedure is also relevant to preparative-scale reactions.

Adopting the optimized conditions described in entry 1 in Table 1, we focused on the generality of this photochemical borylation protocol. The process allows the use of both MIDA and pinacol as chelating agents for boron (Figure 3a). Because of the superior stability of the corresponding adducts, we preferentially used MIDA. However, we note that pinacol in triethylamine can be used in all cases to provide the corresponding pinacol boronic esters of type $3 \mathbf{b}$. As for the radical precursors, this photochemical strategy is suitable for substrates bearing different leaving groups, which can undergo $\mathrm{S}_{\mathrm{N}} 2$ activation from the organic catalyst $\mathrm{A}$. For example, benzyl bromide, iodide, chloride, and mesylate can all be used to deliver the benzyl borylated product $3 c$ (Figure $3 b$ ). In some cases, the activation of benzylic chlorides required the addition of sodium bromide, which was necessary to generate the corresponding bromide in situ (see, for example, products $\mathbf{3 d}$, $3 \mathbf{h}, 3 \mathbf{j}, 3 \mathbf{n})$.

As for the scope of the process (Figure 3c), a wide array of heteroaromatic derivatives can be efficiently functionalized, 
further highlighting the tolerance of this procedure for unprotected polar functional groups. More specifically, this protocol efficiently delivers borylated building blocks featuring a benzotriazole (adduct 3a), thiazole (3d), oxazole (3e), pyrazole (3f), and furan $(\mathbf{3 g})$ moiety. Since this $\mathrm{S}_{\mathrm{N}} 2$-based radical-generation strategy is not reliant on the redox properties of the radical precursors, it could tolerate benzylic substrates bearing electron-donating (product $3 \mathbf{h}$ ) or electronwithdrawing (3i) moieties in the aryl ring. The process is also effective in delivering the desired compounds with high selectivity: an aryl iodide (adduct 3k), an ester (31), and an unprotected alcohol $(3 \mathrm{~m})$ were all tolerated and activated exclusively at the desired benzylic position. In addition to benzylic electrophiles, allyl substrates could be activated because both farnesyl (3n) and prenyl (3o) derivatives could be successfully borylated. As a limitation of the system, alkyl bromides leading to secondary radicals (1p and 1q) or nonstabilized primary radicals (1r) could not be activated, while substrates containing a uracil (1s) or an adenine moiety (1t) afforded the dehalogenated products exclusively. Also pyridine derivatives ( $\mathbf{1} \mathbf{u}$ and $\mathbf{1 v}$ ) did not provide the corresponding borylation products. A complete list of moderately successful and unsuccessful substrates for this radical borylation strategy is reported in section C4, Figure S3, in the Supporting Information.

Cognizant that our $\mathrm{S}_{\mathrm{N}}$ 2-based radical-generation strategy can also produce electrophilic radicals, ${ }^{15}$ we explored the feasibility of implementing a three-component reaction, ${ }^{23}$ where the generated open-shell intermediate is intercepted by an olefin (Figure 4). The newly generated radical would

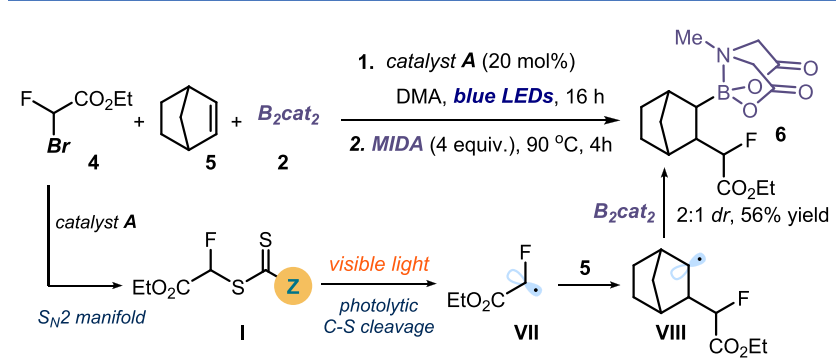

Figure 4. Implementation of a light-driven radical three-component reaction catalyzed by $\mathbf{A}(\mathrm{Z}=$ indole chromophore within $\mathbf{A})$.

then possess the right polarity (nucleophilic radical) to react with $\mathrm{B}_{2} \mathrm{cat}_{2}$, delivering a difunctionalized product. In consonance with this plan, the activation of fluorobromoacetate $\mathbf{4}$ with catalyst A delivered the electrophilic radical VII. A sequential trap with norbornene 5 afforded radical VIII, which could engage in a $\mathrm{C}-\mathrm{B}$ bond formation with $\mathrm{B}_{2} \mathrm{cat}_{2}$. The difunctionalized product 6 was isolated in 56\% yield.

In summary, we have developed a catalytic borylation strategy that uses an easily available organic catalyst and mild reaction conditions, including visible-light illumination and ambient temperature. The chemistry exploits an $\mathrm{S}_{\mathrm{N}}$ 2-based radical-generation strategy, which is not reliant on the redox properties of the radical precursors. Therefore, it grants access to alkyl boronic esters from readily available electrophiles that are difficult to reduce. These include benzylic and allylic chlorides, bromides, and mesylates, which are generally inert to previously reported metal-free borylation protocols. In addition, the redox neutral conditions of this process make it tolerant of redox-sensitive substrates and allow the borylation of heterocyclic-containing substrates.

\section{ASSOCIATED CONTENT}

\section{S Supporting Information}

The Supporting Information is available free of charge on the ACS Publications website at DOI: 10.1021/acscatal.9b01482.

Experimental procedures and spectral data (PDF)

\section{AUTHOR INFORMATION}

\section{Corresponding Author}

*E-mail: pmelchiorre@iciq.es.

\section{ORCID}

Daniele Mazzarella: 0000-0001-7121-9796

Bertrand Schweitzer-Chaput: 0000-0003-2105-6233

Paolo Melchiorre: 0000-0001-8722-4602

\section{Author Contributions}

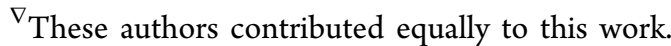

\section{Notes}

The authors declare no competing financial interest.

\section{ACKNOWLEDGMENTS}

Financial support was provided by MICIU (No. CTQ201675520-P), the AGAUR (Grant No. 2017 SGR 981), and the European Research Council (No. ERC-2015-CoG 681840CATA-LUX). D.M. thanks H2020-MSCA-ITN-2016 (No. 722591-PHOTOTRAIN) for a predoctoral fellowship.

\section{REFERENCES}

(1) Brooks, W. L. A.; Sumerlin, B. S. Synthesis and Applications of Boronic Acid-Containing Polymers: From Materials to Medicine. Chem. Rev. 2016, 116, 1375-1397.

(2) Bull, S. D.; Davidson, M. G.; Van den Elsen, J. M. H.; Fossey, J. S.; Jenkins, A. T. A.; Jiang, Y.-B.; Kubo, Y.; Marken, F.; Sakurai, K.; Zhao, J.; James, T. D. Exploiting the Reversible Covalent Bonding of Boronic Acids: Recognition, Sensing, and Assembly. Acc. Chem. Res. 2013, 46, 312-326.

(3) (a) Trippier, P. C.; McGuigan, C. Boronic Acids in Medicinal Chemistry: Anticancer, Antibacterial and Antiviral Applications. MedChemComm 2010, 1, 183-198. (b) Smoum, R.; Rubinstein, A.; Dembitsky, V. M.; Srebnik, M. Boron Containing Compounds as Protease Inhibitors. Chem. Rev. 2012, 112, 4156-4220.

(4) (a) Miyaura, N.; Suzuki, A. Palladium-Catalyzed Cross-Coupling Reactions of Organoboron Compounds. Chem. Rev. 1995, 95, 24572483. (b) Lennox, A. J. J.; Lloyd-Jones, G. C. Selection of Boron Reagents for Suzuki-Miyaura Coupling. Chem. Soc. Rev. 2014, 43, 412-443. (c) Fyfe, J. W. B.; Watson, A. J. B. Recent Developments in Organoboron Chemistry: Old Dogs, New Trick. Chem. 2017, 3, 3155.

(5) Hall, D. G. In Boronic Acids; Hall, D. G., Ed.; Wiley-VCH, 2011; pp $1-133$.

(6) Sandford, C.; Aggarwal, V. K. Stereospecific Functionalizations and Transformations of Secondary and Tertiary Boronic Esters. Chem. Commun. 2017, 53, 5481-5494.

(7) For a review on the topic, see: (a) Kubota, K.; Iwamoto, H.; Ito, $\mathrm{H}$. Formal Nucleophilic Borylation and Borylative Cyclization of Organic Halides. Org. Biomol. Chem. 2017, 15, 285-300. For selected examples, see: (b) Joshi-Pangu, A.; Ma, X.; Diane, M.; Iqbal, S.; Kribs, R. J.; Huang, R.; Wang, C.-Y.; Biscoe, M. R. Palladium-Catalyzed Borylation of Primary Alkyl Bromides. J. Org. Chem. 2012, 77, 66296633. (c) Yang, C.-T.; Zhang, Z.-Q.; Tajuddin, H.; Wu, C.-C.; Liang, J.; Liu, J.-H.; Fu, Y.; Czyzewska, M.; Steel, P. G.; Marder, T. B.; Liu, L. Alkylboronic Esters from Copper-Catalyzed Borylation of Primary and Secondary Alkyl Halides and Pseudohalides. Angew. Chem., Int. 
Ed. 2012, 51, 528-532. (d) Yi, J.; Liu, J.-H.; Liang, J.; Dai, J.-J.; Yang, C.-T.; Fu, Y.; Liu, L. Alkylboronic Esters from Palladium- and NickelCatalyzed Borylation of Primary and Secondary Alkyl Bromides. Adv. Synth. Catal. 2012, 354, 1685-1691. (e) Ito, H.; Kubota, K. Copper(I)-Catalyzed Boryl Substitution of Unactivated Alkyl Halides. Org. Lett. 2012, 14, 890-893. (f) Dudnik, A. S.; Fu, G. C. NickelCatalyzed Coupling Reactions of Alkyl Electrophiles, Including Unactivated Tertiary Halides, to Generate Carbon-Boron Bonds. J. Am. Chem. Soc. 2012, 134, 10693-10697. (g) Bedford, R. B.; Brenner, P. B.; Carter, E.; Gallagher, T.; Murphy, D. M.; Pye, D. R. Iron-Catalyzed Borylation of Alkyl, Allyl, and Aryl Halides: Isolation of an Iron(I) Boryl Complex. Organometallics 2014, 33, 5940-5943. (h) Bose, S. K.; Fucke, K.; Liu, L.; Steel, P. G.; Marder, T. B. Zinccatalyzed Borylation of Primary, Secondary and Tertiary Alkyl Halides with Alkoxy Diboron Reagents at Room Temperature. Angew. Chem., Int. Ed. 2014, 53, 1799-1803. (i) Yoshida, H.; Takemoto, Y.; Kamio, S.; Osaka, I.; Takaki, K. Copper-catalyzed direct borylation of alkyl, alkenyl and aryl halides with $\mathrm{B}(\mathrm{dan})$. Org. Chem. Front. 2017, 4, 1215-1219.

(8) (a) Atack, T. C.; Cook, S. P. Manganese-Catalyzed Borylation of Unactivated Alkyl Chlorides. J. Am. Chem. Soc. 2016, 138, 61396142. (b) Bose, S. K.; Brand, S.; Omoregie, H. O.; Haehnel, M.; Maier, J.; Bringmann, G.; Marder, T. B. Highly Efficient Synthesis of Alkylboronate Esters via $\mathrm{Cu}(\mathrm{II})$-Catalyzed Borylation of Unactivated Alkyl Bromides and Chlorides in Air. ACS Catal. 2016, 6, 8332-8335. A few examples of borylation of alkyl chlorides are also reported in refs $7 \mathrm{c}$ and $7 \mathrm{f}$

(9) (a) Blakemore, D. C.; Castro, L.; Churcher, I.; Rees, D. C.; Thomas, A. W.; Wilson, D. M.; Wood, A. Organic Synthesis Provides Opportunities to Transform Drug Discovery. Nat. Chem. 2018, 10, 383-394. (b) Pitt, W. R.; Parry, D. M.; Perry, B. G.; Groom, C. R. Heteroaromatic Rings of the Future. J. Med. Chem. 2009, 52, 29522963.

(10) For selected examples of metal-catalyzed borylation of heteroarenes, see: (a) Larsen, M. A.; Hartwig, J. F. Iridium-Catalyzed C-H Borylation of Heteroarenes: Scope, Regioselectivity, Application to Late-Stage Functionalization, and Mechanism. J. Am. Chem. Soc. 2014, 136, 4287-4299. (b) Preshlock, S. M.; Plattner, D. L.; Maligres, P. E.; Krska, S. W.; Maleczka, R. E.; Smith, M. R. A Traceless Directing Group for C-H Borylation. Angew. Chem., Int. Ed. 2013, 52, 12915-12919.

(11) For a review in radical borylation methods, see: (a) Yan, G.; Huang, D.; Wu, X. Recent Advances in C-B Bond Formation through a Free Radical Pathway. Adv. Synth. Catal. 2018, 360, 10401053. For examples of radical borylation of aliphatic substrates, see: (b) Hu, D.; Wang, L.; Li, P. Decarboxylative Borylation of Aliphatic Esters under Visible-Light Photoredox Conditions. Org. Lett. 2017, 19, 2770-2773. (c) Jiang, M.; Yang, H.; Fu, H. Visible-Light Photoredox Borylation of Aryl Halides and Subsequent Aerobic Oxidative Hydroxylation. Org. Lett. 2016, 18, 5248-5251.

(12) (a) Candish, L.; Teders, M.; Glorius, F. Transition-Metal-Free, Visible-Light-Enabled Decarboxylative Borylation of Aryl N-Hydroxyphthalimide Esters. J. Am. Chem. Soc. 2017, 139, 7440-7443. (b) Fawcett, A.; Pradeilles, J.; Wang, Y.; Mutsuga, T.; Myers, E. L.; Aggarwal, V. K. Photoinduced Decarboxylative Borylation of Carboxylic Acids. Science 2017, 357, 283-286. (c) Wu, J.; He, L.; Noble, A.; Aggarwal, V. K. Photoinduced Deaminative Borylation of Alkylamines. J. Am. Chem. Soc. 2018, 140, 10700-10704. (d) Sandfort, F.; Strieth-Kalthoff, F.; Klauck, F. J. R.; James, J. M.; Glorius, F. Deaminative Borylation of Aliphatic Amines Enabled by Visible Light Excitation of an Electron Donor-Acceptor Complex. Chem. - Eur. J. 2018, 24, 17210-17214.

(13) Cheng, Y.; Muck-Lichtenfeld, C.; Studer, A. Metal-Free Radical Borylation of Alkyl and Aryl Iodides. Angew. Chem., Int. Ed. 2018, 57, 16832-16836.

(14) Silvi, M.; Melchiorre, P. Enhancing the Potential of Enantioselective Organocatalysis with Light. Nature 2018, 554, 4149.
(15) Schweitzer-Chaput, B.; Horwitz, M. A.; de Pedro Beato, E.; Melchiorre, P. Photochemical Generation of Radicals from Alkyl Electrophiles Using a Nucleophilic Organic Catalyst. Nat. Chem. 2019, 11, 129-135.

(16) Substrates bearing thio functions, including xanthates, have been extensively used in stoichiometric amounts to generate radicals; see: (a) Barton, D. H. R.; Zard, S. Z. Invention of New Reactions Useful in the Chemistry of Natural Products. Pure Appl. Chem. 1986, 58, 675-684. (b) Delduc, P.; Tailhan, C.; Zard, S. Z. A Convenient Source of Alkyl and Acyl Radicals. J. Chem. Soc., Chem. Commun. 1988, 308-310. (c) Zard, S. Z. On the Trail of Xanthates: Some New Chemistry from an Old Functional Group. Angew. Chem., Int. Ed. Engl. 1997, 36, 672-685.

(17) For a study discussing the high nucleophilicity of dithiocarbonate and dithiocarbamate anions of type A, see: Duan, X.-H.; Maji, B.; Mayr, H. Characterization of the Nucleophilic Reactivities of Thiocarboxylate, Dithiocarbonate and Dithiocarbamate Anions. Org. Biomol. Chem. 2011, 9, 8046-8050.

(18) For studies discussing the tendency of thiocarbonyl compounds of type I to generate radicals upon photolytic cleavage, see: (a) Barton, D. H. R.; George, M. V.; Tomoeda, M. Photochemical Transformations. Part XIII. A New Method for the Production of Acyl Radicals. J. Chem. Soc. 1962, 1967-1974. (b) Lalevée, J.; Blanchard, N.; El-Roz, M.; Allonas, X.; Fouassier, J. P. New Photoiniferters: Respective Role of the Initiating and Persistent Radicals. Macromolecules 2008, 41, 2347-2352.

(19) Shaw, M.; Twilton, J.; MacMillan, D. W. C. Photoredox Catalysis in Organic Chemistry. J. Org. Chem. 2016, 81, 6898-6926.

(20) Dag, Ö.; Yaman, S. O.; Onal, A. M.; Isci, H. Spectroelectrochemistry of Potassium Ethylxanthate, Bis(ethylxanthato)nickel(II) and Tetraethylammonium Tris(ethylxanthato)nickelate(II). J. Chem. Soc., Dalton Trans. 2001, 2819-2824. See section F in the Supporting Information for the redox properties of catalyst $\mathrm{A}$.

(21) An alternative radical self-propagating mechanism, based on a single-electron transfer (SET) between radical VI and intermediate I (when $\mathrm{R}=\mathrm{Ph}, \mathrm{E}_{\text {red }}\left(\mathrm{I} / \mathrm{I}^{\bullet}\right)=-1.5 \mathrm{~V}$ vs $\mathrm{Ag} / \mathrm{AgCl}$ ) cannot be excluded. Other mechanistic possibilities are less likely. For example, we did not observe any electron-donor acceptor (EDA) complex formation between a preformed intermediate $I$ and $B_{2} c_{2} t_{2} 2$. In addition, a radical chain mechanism involving an SET between intermediate VI and difficult-to-reduce alkyl chlorides/sulfonates substrates can be excluded based on their reduction potentials. Those substrates are also not prone to atom transfer manifolds. See section E in the Supporting Information for more details.

(22) Lu, D.; Wu, C.; Li, P. Synergistic Effects of Lewis Bases and Substituents on the Electronic Structure and Reactivity of Boryl Radicals. Chem. - Eur. J. 2014, 20, 1630-1637.

(23) Cheng, Y.; Muck-Lichtenfeld, C.; Studer, A. Transition MetalFree 1,2-Carboboration of Unactivated Alkenes. J. Am. Chem. Soc. 2018, 140, 6221-6225. 